\title{
Panorama de la Gestión de Activos para Transformadores de Potencia
}

\author{
Andrés F. Cerón ${ }^{(1)}$, Iván F. Orduña(1), Guillermo Aponte ${ }^{(1)}$, Andrés A. Romero(2) \\ (1) Grupo de Investigación en Alta Tensión GRALTA. Universidad del Valle, Ciudad Universitaria Meléndez, \\ Calle 13 No 100-00, Cali - Colombia (e-mail: andres.ceron@correounivalle.edu.co). \\ (2) Instituto de Energía Eléctrica IEE UNSJ-CONICET. Universidad Nacional de San Juan - CONICET, \\ Av. Libertador General San Martín 1109 (Oeste), San Juan - Argentina. (e-mail: aromero@iee.unsj.edu.ar).
}

Recibido Jun. 25, 2014; Aceptado Sep. 8, 2014; Versión final recibida Nov. 7, 2014

\section{Resumen}

Este artículo presenta una revisión general del marco conceptual relacionado con la gestión activos para transformadores de potencia y la evaluación del riesgo asociado a sus fallas. El índice de riesgo es una herramienta esencial para realizar la gestión de activos, especialmente porque apoya al gestor en el proceso de toma de decisiones. El índice de riesgo está compuesto por dos factores: i) el factor de consecuencia, que valora los impactos que se podrían presentar en caso de falla del equipo; y ii) la probabilidad de falla que se determina a partir de los resultados de las pruebas de funcionamiento, de la historia de la operación y del historial de fallas, entre otros aspectos. Se presentan aspectos generales relacionados con la gestión de activos según las normas internacionales, para luego tratar con mayor detalle aspectos específicos relacionados con la evaluación del índice de riesgo del transformador, encontrados en la literatura.

Palabras clave: transformador de potencia, gestión de activos, índice de riesgo, ensayos de campo

\section{Overview of Asset Management for Power Transformers}

\begin{abstract}
This article presents an overview of the conceptual framework related to the asset management of power transformers, and to the assessment of risks associated with transformer failures. The risk index is an essential tool for developing the management of this asset, especially because it supports the asset manager in the decision-making process. The risk index is composed by two indicators: i) consequence factor, which assesses the impacts that could arise in the event of equipment failure, and ii) the probability of failure, which is determined from the results of tests for transformer functioning, operating history, and failure history, among others. General aspects of asset management according to international standards are presented, to then deal with specific aspects related to evaluation of the transformer risk index, found in the literature.
\end{abstract}

Keywords: power transformer, asset management, risk index, laboratory tests 


\section{INTRODUCCIÓN}

La sobrecarga en la capacidad nominal de los transformadores, la reducción en gastos de mantenimiento y la austeridad en la inversión, son los desafíos que deben enfrentar las compañías eléctricas en la actualidad (Bartley, 2013). Como respuesta a estos requerimientos, las compañías eléctricas están introduciendo métodos usados por los inversionistas y las compañías de seguros, relacionados con el aseguramiento de los activos y la minimización de los riesgos (Durán, 2013). En vista del gran número de transformadores en el mundo que se acercan al final de su vida útil (Energy D., 2014), las compañías eléctricas están interesadas en identificar el tiempo de vida remanente y la condición de los mismos (Mariño et al., 2004), tratando así de extender al máximo su vida útil o tomar decisiones oportunas que minimicen costos, producto de una eventualidad y de esta forma maximizar los beneficios económicos, producto de la explotación del activo (Abu Elanien y Salama, 2010).

Una compañía eléctrica estará preparada para abordar los retos planteados, sólo si cuenta con una metodología para la gestión óptima de sus activos, es decir, si puede tomar las decisiones correctas en el momento adecuado (Romero et al., 2012). La gestión de un parque de transformadores de potencia debe tener en cuenta los riesgos asociados a cada transformador dentro del sistema eléctrico. En este sentido, se debe valorar el nivel de riesgo para cada unidad. Al valorar el riesgo, se puede establecer una clasificación por mérito y condición de los transformadores, lo que permite la planificación de acciones para el mediano y largo plazo.

La gestión de activos se define como el conjunto de actividades y prácticas a través de las cuales una organización empresarial maneja de forma óptima y eficiente sus activos con el propósito de alcanzar un plan estratégico organizacional. En este contexto la palabra "activo" se refiere a la planta, maquinaria, propiedades, edificios, vehículos y otros elementos de valor importante para cualquier organización empresarial. El propósito de este artículo es mostrar un panorama de la gestión de activos aplicada en transformadores de potencia.

La implementación de un sistema de gestión de activos debe estar enmarcado dentro de los siguientes principios (Reetz, 2005): Holístico: ser multi-disciplinario y enfocado en todos los puntos de vista de los activos; ii) Sistemático: aplicarse de manera rigurosa en un sistema de gestión estructurado; iii) Sistémico: los activos deben cuidarse desde un punto de vista global, observando todos los elementos que agregan o restan valor y no con visiones particulares; iv) Basado en riesgo: la evaluación de riesgo debe estar presente en toda la toma de decisiones y planes sobre los activos; v) Óptimo: tener métodos claros para obtener el mejor beneficio de los activos para la organización; vi) Sustentable: la gestión debe cubrir el ciclo de vida total de los activos desde el diseño hasta la desincorporación, considerando la edad de los mismos, el deterioro con el tiempo, opciones de renovación, mejoramiento; y vii) Integrado: integrar los intereses y obligaciones de todas las partes que juegan un papel en la gestión de los activos, esto cubre desde accionistas, trabajadores, clientes, reguladores, etc.

\section{GESTIÓN DE ACTIVOS}

En los años 90, aparece el concepto de la gestión de activos dentro de la producción petrolera del mar del norte. Este concepto generó excelentes beneficios económicos y además generó muchas interpretaciones para el concepto gestión de activos pasando por las finanzas, la informática, el mantenimiento, los bienes raíces. Ante esta situación, el Institute of Asset Management (IAM) en colaboración con el British Standards Institute (BSI), estableció en el año 2002, la norma BSI PAS 55 denominada "Asset Management", la cual fue acogida de inmediato por muchas organizaciones empresariales que vieron su gran potencial.

En el año 2006, se generó un sistema de certificación que fue de carácter de obligatorio en los segmentos de energía y gas en el Reino Unido. En el año 2008, surgió una nueva versión de la norma (BSi PAS 55, 2008). La norma BSI PAS 55, cubre todas las etapas en el ciclo de vida de un activo, desde la ingeniería, operación, mantenimiento hasta la renovación de los mismos (Durán, 2013). Finalmente, en febrero de 2014, se publica la serie de normas internacionales ISO 55000/1/2 (2014), las cuales permiten a una organización lograr sus objetivos a través de la gestión eficaz y eficiente de sus activos, y de manera consistente y sostenible en el tiempo.

\section{Tipos de activos}

La norma BSi PAS 55 (2008), está dirigida a los activos físicos, pero implícitamente toca algunos aspectos de los restantes activos definidos del ámbito empresarial, tales como los recursos humanos, los intangibles, los financieros y la información (ver Fig. 1). 


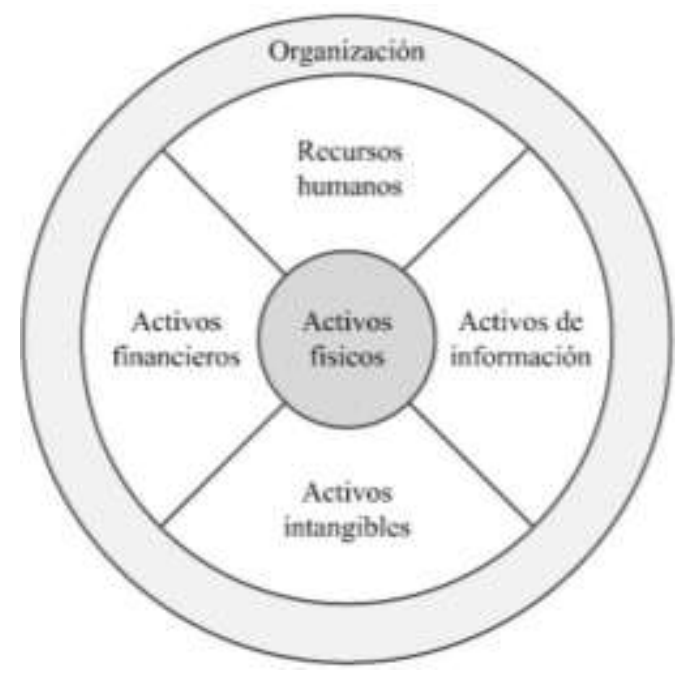

Fig. 1. Tipos de activos (Bsi PAS 55, 2008)

\section{Estructura de un sistema de gestión de activos}

El propósito de concebir un sistema de gestión de activos es apoyar la ejecución del plan estratégico de una organización empresarial, con el fin de satisfacer las expectativas técnico-económicas de una gran variedad de partes interesadas (Hardwick, 2007). El plan estratégico de la organización es el punto de partida para el desarrollo de las políticas, estrategias, objetivos y planes de gestión de activos (ver Fig. 2).

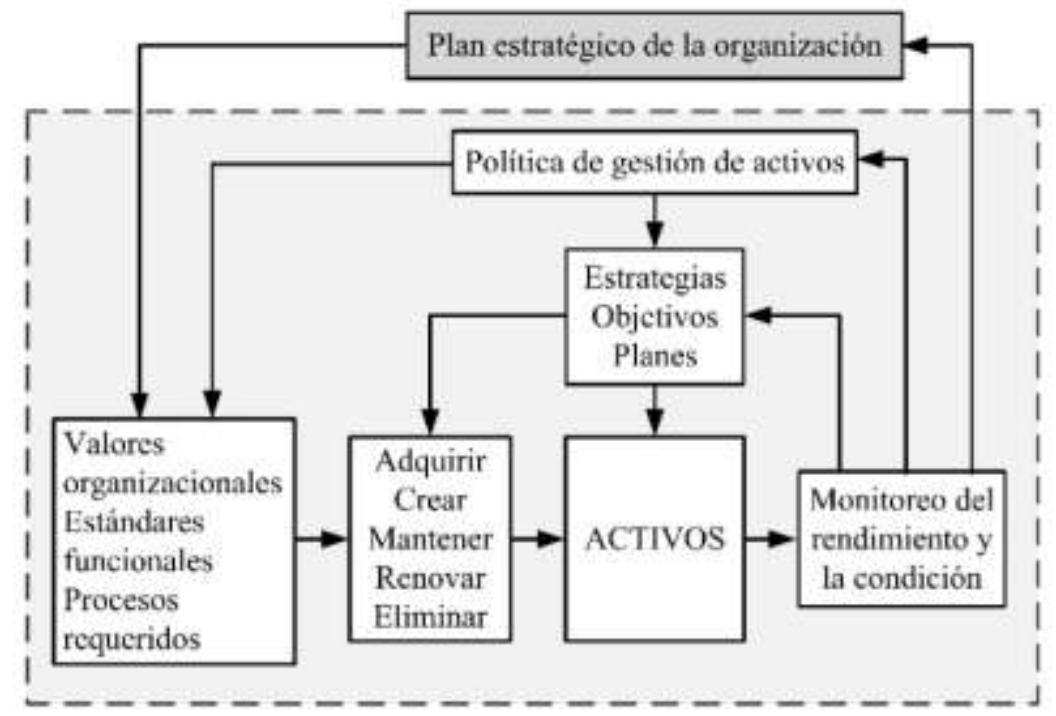

Fig. 2. Sistema de gestión de activos (Bsi PAS 55, 2008)

\section{GESTIÓN DEL TRANSFORMADOR DE POTENCIA}

En la actualidad hay más de 100.000 transformadores de potencia que operan en los Estados Unidos y más de 400.000 en todo el mundo (Energy D., 2014). Este es un equipo de capital intensivo y puede alcanzar hasta el $60 \%$ del total de la inversión de una subestación, además es fundamental para la operación de cualquier sistema de potencia (Naderian et al., 2009). El transformador es una máquina eficiente y confiable, que en condiciones normales de operación, está diseñada y construida para tener una vida útil cercana a los 40 años (Romero et al., 2012).

Al igual que las personas, el transformador se ve afectado por el paso del tiempo, por esta razón, a medida que se hace más longevo, se incrementa su probabilidad de falla. De acuerdo a Hartford Steam Boiler, una de las mayores aseguradoras de transformadores de los Estados Unidos, 3 de cada 100 transformadores de una gran cuantía instalada en la década de 1960, están propensos a fallas (Tichelman, 2004). El costo promedio de reemplazo de un transformador de 100 MVA puede alcanzar los US\$2 millones y puede tomar de 18 a 24 meses su construcción (Energy D., 2014). 
Durante todo el ciclo de vida de un transformador de potencia se pueden realizar algunas acciones como: 'adquirir', 'mantener', 'reparar', 'reubicar', 'desechar', 'reemplazar' o 'no hacer nada'. Los expertos afirman que alrededor del $53 \%$ de las fallas de los transformadores se pueden prevenir (Tichelman, 2004); p.ej., antes de generar una falla, el transformador produce gases, por lo tanto su detección temprana podría ser clave para evitar una contingencia futura.

El gestor de este tipos de activos, se enfrenta a inquietudes como: ¿Dónde y cuándo se debe tomar una acción?, ¿Cuál de las acciones es la mejor?, ¿Cuál es el costo económico de cada acción?, ¿Cuáles son las consecuencias de cada acción?, etc. En la Fig. 3, se muestran las probables acciones a seguir en la gestión del activo transformador de potencia.

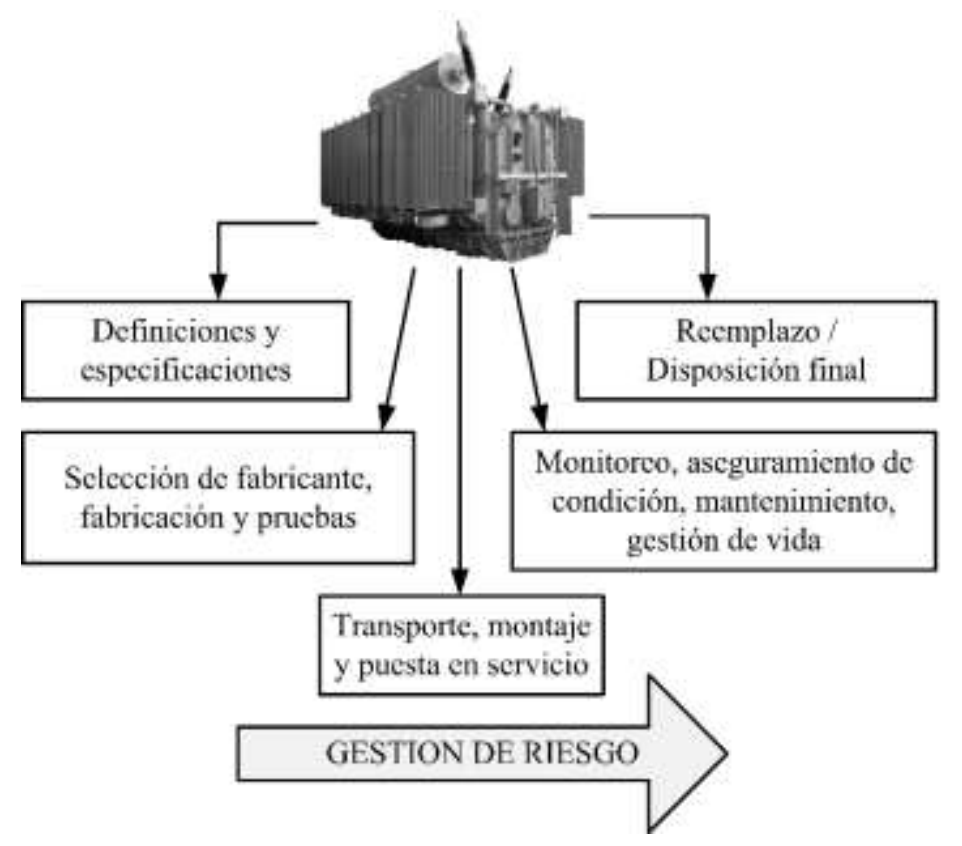

Fig. 3. Acciones en la gestión del activo transformador

Para realizar cualquier acción sobre un transformador de potencia se debe contar con suficiente información y una metodología de actuación eficiente. Cada decisión sobre el transformador tiene un riesgo asociado y cada acción un costo; por lo tanto, resulta clave valorar los riesgos y los costos en todos los probables escenarios.

\section{Valoración del riesgo}

Un índice de riesgo es un indicador útil para la toma de decisiones estratégicas sobre un activo (BSi PAS 55, 2008). El índice de riesgo se determina a partir de dos componentes: a) El factor de consecuencia (FC): es un análisis económico, basado en la premisa de que todo activo fallará en el futuro; y b) La probabilidad de falla (PF): evalúa la condición técnica del activo mediante ensayos de diagnóstico, historial de carga, historial de eventos, índice de salud, etc. Una vez se calculan los factores FC y PF, estos son graficados en una matriz de riesgo, como la de la Fig. 4. Se observa que la matriz de la Fig. 4 está definida en términos de variables lingüísticas. No obstante, las escalas tanto para el FC y el PF pueden también darse en términos de valores numéricos. En general la valoración varía de acuerdo con las metodologías seleccionadas para determinar los índices, por ejemplo en Ashkezari et al. (2013) se presenta una metodología para determinar un índice de salud del aislamiento del transformador (relacionado con PF) el cual toma valores de uno (1) para una condición excelente o probabilidad de falla muy baja y cinco (5) para una condición insatisfactoria o probabilidad de falla alta. Otro ejemplo se da en Lara y Liñan (2009) quienes presentan una metodología para valorar el estado del transformador entregando a la salida un indicador de condición que varía entre cero (0) y trescientos (300) y permite identificar tres categorías de estado, de hecho: Cat. I entre 0 y 100 indica un funcionamiento normal y no requiere acción; Cat. II entre 100 y 200 indica condición regular e implica realizar una inspección inmediata; y Cat. III entre 200 y 300 indica alta probabilidad de falla e implica sacar de servicio la máquina y realizar acciones de reparación inmediatas. Un último ejemplo se presenta en Abu Elanien et al. (2012) quienes presentan una metodología para valorar el índice de salud de un transformador, el cual varía entre cero (0) que representa una condición muy buena o probabilidad de falla muy baja y uno (1) para una condición muy mala o probabilidad de falla muy alta. A continuación se 
describirán los principales componentes que se deben considerar para valorar cada uno de los dos índices que componen el índice de riesgo.

Factor de consecuencia (FC)

El factor de consecuencia para cada transformador varía según su tamaño y posición en el sistema eléctrico, su carga asociada, etc. Algunas de las variables consideradas para el cálculo de este factor son: Costos asociados durante el ciclo de vida (CCV), Confiabilidad del sistema, Factor de redundancia, Seguridad del sistema e Impacto ambiental y seguridad pública:

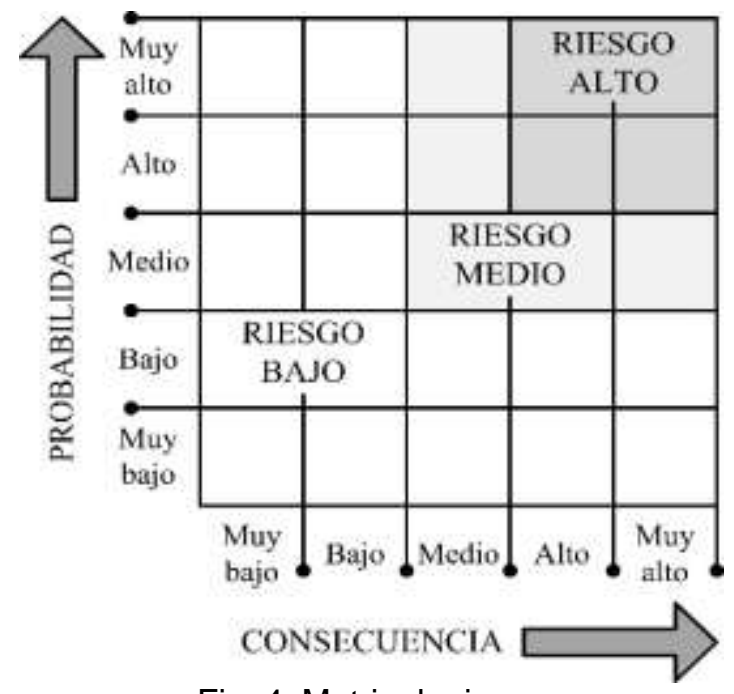

Fig. 4. Matriz de riesgo

Costos asociados durante el ciclo de vida (CCV): La evaluación de costos es necesaria cuando se deben comparar diferentes opciones de inversión. Los costos se pueden clasificar de acuerdo a la etapa en que el transformador se encuentra en su ciclo de vida (IEC 60300-2, 2004): p.ej., costos de adquisición (CA), costos de administración, operación y mantenimiento (COM) y costos de disposición y reciclaje (CD). Los costos durante el ciclo de vida del transformador (CCV) se pueden definir como (Romero et al., 2012):

$$
C C V(t)=C_{A}(t)+C_{O M}(t)+C_{D}(t)
$$

El costo CA es considerado cuando la posibilidad de una inversión se está planteando, ya sea por adquisición, reparación de una unidad fallada o instalación de otra unidad dentro de una subestación. El costo COM se relaciona con las estrategias de mantenimiento a aplicar sobre el activo. Los diferentes tipos de mantenimiento se pueden clasificar en: mantenimiento correctivo, mantenimiento basado en el tiempo, mantenimiento basado en la condición y mantenimiento centrado en la confiabilidad. El mantenimiento debe incluir los costos relacionados con la mano de obra, suministros, equipos especializados, pruebas de laboratorio y consultoría de expertos. En cuanto a los CD, se deben considerar los costos por recolección, transporte, tratamiento y disposición final de residuos, p.ej., el aceite. Por otro lado los beneficios recibidos por el reciclado, p.ej., el cobre y el hierro también inciden en los CD, que de hecho deben sustraerse. Es importante observar que al momento de evaluar las consecuencias de la falla final del transformador, los costos que tienen mayor incidencia son los de adquisición y los de disposición. Es así como en la actualidad, el costo de adquisición o reparación resulta ser el principal factor a considerar para el reemplazo de la unidad, por lo que resulta difícil justificar tomar la decisión de su reemplazo antes de que ocurra la falla final (Romero et al., 2012).

Confiabilidad del sistema: Las interrupciones del suministro de energía eléctrica son debidas a la salida de uno o varios componentes de la red. Aunque, no siempre una salida trae consigo una interrupción. Cuantificar la confiabilidad de un sistema eléctrico requiere de índices de confiabilidad. Estos índices deben ser definidos de forma estándar con el propósito de que sean comparables, reproducibles e interpretables. Uno de los índices más reconocidos para la evaluación de la confiabilidad es la energía no suministrada (ENS), el cual se determina a partir de la sumatoria de la potencia activa no suministrada multiplicada por el tiempo de duración de cada interrupción ( $\mathrm{Li}, 2005)$. Otros índices de confiabilidad están asociados al número de clientes afectados por cada interrupción (customer average interruption duration index), la duración de cada interrupción (system average interruption duration index) y la cantidad de interrupciones (system average interruption frequency index) (Eto y LaCommare, 2008). Como parte del análisis de confiabilidad también se deben evaluar los costos sociales asociados con la falla del transformador, por lo tanto es necesario identificar y clasificar los diferentes tipos de clientes conectados a la red de suministro 
eléctrica, p.ej., clientes residenciales, comerciales, industriales y agrícolas. Es necesario entonces conocer la visión del cliente en lo que se refiere al producto de la energía eléctrica, sus usos y los impactos en la producción. Los costos de no tener electricidad no sólo dependen del tamaño del consumo, sino también de los costos de no poder desarrollar una actividad productiva, existen varios métodos para evaluar el costo de las interrupciones, pero en general pueden ser agrupados en: métodos directos e indirectos. En los métodos directos la información de los costos es solicitada al cliente, para luego establecer una relación entre la falta de la energía y los daños causados en la producción, la pérdida de las ventas en el comercio, etc. En los métodos indirectos se usa el principio económico de la sustitución: el valor del producto sustituto es una medida del valor del producto original. Es decir, el costo que el cliente está dispuesto a asumir para evitar la interrupción (p. ej. instalación de UPS, generador, entre otros).

Factor de redundancia: Las redes de sub-transmisión y de transmisión de energía eléctrica, normalmente están configuradas en anillo; por consiguiente existen ramas alternativas para transmitir la energía. En condiciones normales de operación, la corriente eléctrica fluye a través de dos o más ramas de la red. Si sucede un corte en alguna de las ramas, la o las ramas restantes asumirán la carga de la rama interrumpida. En esta situación los clientes no experimentarán ninguna interrupción. Esto se conoce como "redundancia". La redundancia, mejora significativamente la continuidad del suministro pero puede aumentar significativamente los costos de las inversiones en el sistema eléctrico (transformadores en paralelo, unidades móviles, interconexiones, entre otros.) (Romero et al., 2012).

Seguridad del sistema: Un criterio para la evaluación de la seguridad de un sistema eléctrico es el principio determinista $(\mathrm{N}-1)$. El objeto de este principio es garantizar que la salida de un elemento fundamental de un sistema de potencia pueda ser soportada sin afectar la confiablidad del sistema. Bajo este principio, un sistema eléctrico en estado de contingencia debe continuar operando sin sobrepasar los límites de seguridad de los elementos (líneas, transformadores de potencia, etc.). En algunos casos, se busca el cumplimiento de criterios (N-2) o (N-3) debido a que durante la operación normal de un sistema eléctrico, se pueden tener situaciones en las que un elemento falle mientras que otros estén fuera de servicio por labores de mantenimiento.

Impacto ambiental y seguridad pública: Considera la cantidad de litros de aceite que podrían impactar al medio ambiente en caso del siniestro de un transformador de potencia y el costo de su limpieza. Por otro lado, también debe considerar viviendas próximas a la estación transformadora, equipamientos y personal de la subestación, que podrían ser afectadas en tal situación (explosión, incendio, entre otros.).

Para obtener el factor consecuencia deben integrarse los aspectos antes mencionados, teniendo en cuenta que las consecuencias en general pueden medirse en términos monetarios, el FC se puede calcular a través de una función de costo (Romero et al., 2012):

$$
F C=f(C C V, E N S, S, I A)
$$

Cada parámetro de f se puede obtener a través de un sub-modelo de costo. CCV es el costo durante todo el ciclo de vida, ENS es el costo de la energía no suministrada durante una interrupción, S es el costo de la penalización por la insuficiencia en el servicio e IA es el costo del impacto ambiental.

\section{Probabilidad de falla (PF)}

Antes de introducir el índice de Probabilidad de Falla (PF), se debe observar que un transformador de potencia es una maquina compuesta por varios subsitemas, cuya condición puede ser monitoreada y evaluada de forma independiente. A pesar de que estos subsistemas interactúan entre sí y pueden aportar a la falla del sistema general, se debe considerar que algunos subsistemas son de fácil mantenimiento con respecto a otros, a los que el acceso es limitado o incluso prohibitivo durante la normal operación de la máquina; por lo que tales subsistemas resultan ser de mayor criticidad en la determinación de la probabilidad de falla final de una unidad transformadora. Varios trabajos han identificado de forma exitosa los principales subsistemas de un transformador, pero uno de los más completos está desarrollado en IEEE C57.140 (2006), donde el transformador es dividido en 8 subsistemas principales y 25 componentes (Tabla 1).

En la Tabla 1 se observa que de los ocho subsistemas, dos de ellos componen la parte activa de la unidad, es decir, el núcleo y los devanados. Son justamente estos dos subsistemas los de más difícil mantenimiento ya que los mismos conforman la parte interna del equipo, están sumergidos en el aceite, no son reemplazables fácilmente y una intervención implica desencubar la unidad, acción de elevado riesgo e indeseable por parte de los propietarios del activo puesto que facilita el ingreso de humedad, implica largos tiempo de fuera de servicio, puede producir pérdidas y vertimientos de aceite, entre otras consecuencias. La situación difiere con los otros subsistemas (pasatapas, sistema conservador de aceite, sistema de 
refrigeración, cambiadores de derivaciones y parte externa del tanque) ya que estos el estar ubicados en el exterior de la máquina, son susceptibles a mantenimientos más frecuentes y menos riesgosos y costosos, e incluso a la sustitución completa del subsistema (obviamente con la excepción del tanque). Luego, si se realiza un mantenimiento y monitoreo adecuado de los otros seis subsistemas, se puede evaluar la probabilidad de falla del transformador bajo la premisa de que el fin de vida del transformador se alcanzará cuando fallen alguno de los dos subsistemas núcleo y devanado, los que además tienen como componente crítico al papel aislante.

Tabla 1. División del Transformador de Potencia (IEEE C57.140, 2006)

\begin{tabular}{|c|c|c|c|c|c|c|c|c|c|c|c|c|}
\hline Subsistema & Componente & 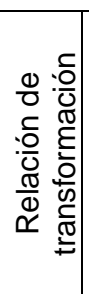 & 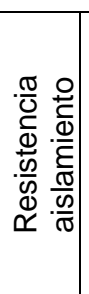 & 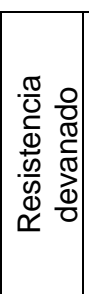 & 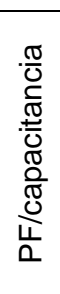 & 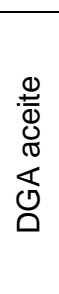 & $\begin{array}{l}\overline{\sigma 0} \\
\overline{00} \\
; 0\end{array}$ & 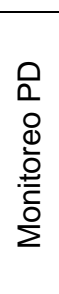 & 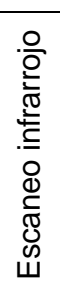 & 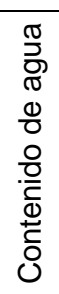 & 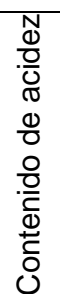 & 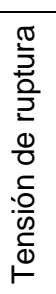 \\
\hline \multirow{3}{*}{$\begin{array}{l}\text { Pasatapa } \\
\text { (Bushing) }\end{array}$} & Condensador & & & & $\mathrm{X}$ & & & & & & $\mathrm{X}$ & $X$ \\
\hline & Aceite & & & & $\mathrm{X}$ & $X$ & $X$ & & & $\mathrm{X}$ & & \\
\hline & Escudo corona & & & & & $\mathrm{X}$ & & $\mathrm{X}$ & & & & \\
\hline \multirow{2}{*}{ Devanado } & Conductor/devanado & $x$ & $\mathrm{X}$ & $x$ & $x$ & $x$ & & $X$ & & & & \\
\hline & Aislamiento & $X$ & $x$ & $X$ & $\mathrm{X}$ & $X$ & & $X$ & & & $\mathrm{X}$ & $X$ \\
\hline \multirow{2}{*}{ Núcleo } & Núcleo de acero & & & & & $\mathrm{X}$ & & & & & & \\
\hline & Aislamiento & & $\mathrm{X}$ & & & $\mathrm{X}$ & & $\mathrm{X}$ & $\mathrm{X}$ & & $X$ & $\mathrm{X}$ \\
\hline \multirow{3}{*}{$\begin{array}{l}\text { Sistema } \\
\text { conservador del } \\
\text { aceite }\end{array}$} & Aceite & & $\mathrm{X}$ & & $X$ & $X$ & & & $\mathrm{X}$ & $\mathrm{X}$ & & \\
\hline & Conservador & & & & $\mathrm{X}$ & $\mathrm{X}$ & $X$ & & & & $X$ & $X$ \\
\hline & Tubería & & & & & & $\mathrm{X}$ & & & & & \\
\hline \multirow{5}{*}{ Refrigerador } & Bomba & & & & & $\mathrm{X}$ & $X$ & & & & & \\
\hline & Aspas & & & & & $x$ & $x$ & & & & & \\
\hline & Placas del radiador & & & & & & $X$ & & & & & \\
\hline & Placas tubulares & & & & & & $X$ & & & & & \\
\hline & Carcasa & & & & & & $x$ & & & & & \\
\hline \multirow{2}{*}{$\begin{array}{lr}\text { Cambiador } & \text { de } \\
\text { derivaciones } & \text { des- } \\
\text { energizado (DETC) }\end{array}$} & Contactos & & & & & $\mathrm{X}$ & & & & & & \\
\hline & Árbol de transmisión & & & & & & $\mathrm{X}$ & & & & & \\
\hline \multirow{5}{*}{$\begin{array}{lr}\text { Cambiador } & \text { de } \\
\text { derivaciones } & \text { bajo } \\
\text { carga (LTC) } & \end{array}$} & Contactos & & & & & $\mathrm{X}$ & & & & & & \\
\hline & Árbol de transmisión & & & & & & $\mathrm{X}$ & & & & & \\
\hline & Circuito de control & & & & & & $\mathrm{X}$ & & & & & \\
\hline & Tablero aislador & & & & & $\mathrm{X}$ & & & & & & \\
\hline & Empaques & & & & & $\mathrm{X}$ & & & & & & \\
\hline \multirow{3}{*}{ Tanque } & Empaques & & & & & $x$ & & & & & & \\
\hline & Tubería & & & & & $\mathrm{X}$ & & & & & & \\
\hline & Estructura de acero & & & & & $X$ & $\mathrm{X}$ & & $\mathrm{X}$ & & & \\
\hline
\end{tabular}

En el anterior contexto, la estimación del índice de probabilidad de falla se realiza en función de los resultados y el análisis de condición observado a partir de las pruebas relacionadas con la parte activa de la unidad y su respectivo sistema de aislamiento. Para determinar PF es necesario adquirir información (monitoreo de la condición) y luego procesarla (diagnóstico de la condición). En la actualidad existen varias técnicas para evaluar la condición de la parte activa de los transformadores de potencia, adicionalmente, se adelantan investigaciones para mejorarlas. A continuación se presentarán brevemente algunas de estas técnicas.

Envejecimiento del aislamiento en base a la curva de carga: con esta herramienta se busca determinar la vida efectiva de la celulosa de los devanados en función de parámetros característicos del transformador, datos históricos de la curva de carga y de la temperatura ambiente, debido a que en condiciones normales de operación, el envejecimiento del papel corresponde con el del transformador de potencia. La celulosa se envejece con mayor celeridad en el punto más caliente del bobinado (hot-spot winding temperature, HST). Sin embargo, para hallar el HST se presenta la dificultad de falta de información detallada de la curva de carga y de la temperatura ambiente, la cual se recomienda debería adquirirse, como mínimo, en intervalos de 3 minutos. Cuando los intervalos de tiempo son mayores se puede subestimar o sobreestimar el envejecimiento del aislamiento, debido a cambios bruscos en el perfil de carga, situación que puede conducir a errores acumulados intolerables. Para tratar con este problema, actualmente se investiga la preinstalación (en fábrica) de medidores de temperatura basados en fibra óptica, los cuales se colocan directamente sobre los puntos de interés, para luego correlacionar los incrementos de temperatura con el 
control de las diferentes etapas de refrigeración y, adicionalmente, calcular con mayor exactitud la degradación del papel (Bérubé et al., 2009).

Análisis del histórico y registro de fallas: Los registros de datos de la historia de reparación y mantenimiento pueden indicar los incidentes de carácter aislado o recurrente. Documentación de las inspecciones de campo e informes de mantenimiento del transformador deben ser revisados, ya que pueden indicar la necesidad de una mayor frecuencia de mantenimiento para una condición sospechosa. Los registros de fallas en, o cerca del transformador son indicadores de estrés que los bobinados han experimentado. Estos incidentes incluyen fallas de los pasatapas, problemas y fallas con el cambiador de derivaciones bajo carga, fallas en interruptores, entre otras. Todas estas perturbaciones tienen un efecto acumulativo en los bobinados del transformador (Bartley, 2013).

Condiciones de trabajo: La frecuencia y el tipo de mantenimiento, el ajuste de las protecciones contra sobrecarga y cortocircuito, la adecuación de las protecciones contra sobretensiones, las condiciones ambientales (temperatura ambiente, humedad, contaminación, etc.), entre otros factores de trabajo influyen en la vida útil del transformador, luego el adecuado registro de este tipo de información es necesaria para realizar el seguimiento de la vida útil del transformador. De igual forma, las condiciones de almacenamiento también deben ser tomadas en consideración (Bartley, 2013).

Fabricante y año de fabricación: Esta información, puede ser indicativa de la calidad del transformador, el material y el estado de los componentes. La fecha de fabricación además de indicar la edad calendario de la máquina, puede dar indicios de las tecnologías empleadas para la fabricación del equipo. Por ejemplo, el aislamiento de celulosa térmicamente mejorado no estaba disponible antes de 1970, y se debe notar que los transformadores fabricados con papel no mejorado son más susceptibles a la degradación del aislamiento por alta temperatura y humedad (Bartley, 2013).

Grado de polimerización (DP): Mide la resistencia mecánica del aislamiento solido (papel) del transformador. Este valor representa el número de monómeros $\beta$ de glucosa presentes en las moléculas de celulosa. En el momento de la fabricación de un transformador de potencia el DP se encuentra usualmente entre 1000 y 1300 pero con el tiempo este valor va disminuyendo gradualmente. En general se considera que si el valor de DP es menor que 200, entonces el papel ha perdido sus propiedades mecánicas y por lo tanto el transformador es susceptible a una falla destructiva (Lundgaard et al., 2004). La principal desventaja de esta técnica de evaluación del estado del aislamiento sólido, es que la unidad debe estar fuera de servicio y tiene que ser abierta para extraer la muestra de papel, exponiendo así el transformador al ingreso de humedad (Romero et al., 2012).

Análisis de gases disueltos (DGA): Un transformador de potencia en condiciones normales de operación genera gases. Sin embargo, la concentración de estos gases se puede incrementar debido a la presencia de una anormalidad o falla en el interior del transformador y por envejecimiento del mismo (IEEE C57.104, 2008). El aceite genera gases como acetileno, hidrógeno, metano, etileno y etano, mientras que la celulosa o papel genera gases derivados del carbono (Abu Elanien et al., 2012). La evaluación de la concentración de estos gases se realiza a través del DGA. Existen varias metodologías para interpretar sus resultados: p.ej., las relaciones de Dornenburg, las relaciones de Rogers, las relaciones IEC, el triángulo de Duval, el método del gas característico y la concentración total de gases. Las grandes ventajas del DGA son que no es necesario desconectar el transformador mientras se evalúa su condición (evaluación en línea), y que es posible identificar fallas incipientes (IEEE C57.104, 2008; IEC 60599, 2007).

Análisis de vibraciones: El uso de vibraciones para la evaluación de la salud de un transformador es una técnica relativamente nueva en comparación con otros métodos de diagnóstico. Las vibraciones del transformador, se componen de las vibraciones en el núcleo, en los devanados y en el cambiador de derivaciones bajo carga (OLTC) (Shengchang et al., 2001). Estas vibraciones, se propagan a través del aceite del transformador hasta llegar a las paredes del tanque en el cual se pueden registrar mediante sensores de vibración. El análisis de vibraciones es una buena herramienta para la evaluación de la salud del OLTC (Kang y Birtwhistle, 2001).

Análisis de la respuesta en frecuencia (FRA): Cuando un transformador se somete a elevadas corrientes de falla, los devanados son sometidos a severos esfuerzos mecánicos que pueden causar movimientos, deformaciones y en algunos casos daños graves. Estas deformaciones pueden generar cambios en la inductancia y en la capacitancia característica del devanado. La detección de daños puede llevarse a cabo mediante la comparación de una onda de FRA de un devanado sano contra otra onda de FRA de un devanado dañado. Los cambios en la onda se pueden utilizar para estimar el grado de daño del devanado y su posible ubicación (Aponte et al., 2011). 
Descargas parciales (PDs): En los materiales dieléctricos existen imperfecciones y cuando el material aislante es sometido a una diferencia de potencial, el campo eléctrico tiende a concentrarse en tales imperfecciones, produciendo ionización y dando origen a PDs que afectan principalmente las zonas de imperfección. Con el tiempo estas imperfecciones pueden terminar deteriorando todo el sistema de aislación del transformador de potencia (Wang et al., 2002). Hay dos métodos utilizados para la detección de PDs: La detección de las señales acústicas y la medición de las señales eléctricas (Cardoso y Oliveira, 1999). Las PDs pueden ser medidas a través de sensores piezoeléctricos, fibra óptica y sensores de extra-alta frecuencia (Zargari y Blackburn, 1996). También pueden ser detectadas indirectamente usando técnicas químicas como la medición de los productos de degradación producidos por las PDs (Wang et al., 2002).

Contenido de furanos: El deterioro de la estructura química del papel como consecuencia de una mayor temperatura de operación del transformador y un alto contenido de humedad, disminuye el valor del grado de polimerización (DP) lo cual es proporcional a un mayor contenido de compuestos furánicos disueltos en el aceite, especialmente del compuesto 2-furaldehido cuya proporción es más del $90 \%$ comparada con la de los demás furanos. El contenido de 2-furaldehido (2-FAL) se considera el factor más importante en la evaluación de la salud del transformador, ya que evalúa directamente la salud del aislamiento sólido (IEC 61198, 1993).

A partir de información obtenida de muestras de laboratorio y de transformadores fuera de servicio, ya sea para reparación o investigación, se han desarrollado modelos matemáticos que relacionan la concentración del compuesto furanico (2FAL) con el valor de DP (Sidwell, 2011). Uno de los primeros modelos fue desarrollado con base en datos recolectados de transformadores con papel normal kraft (ver Ecuación 3 ).

$$
D P=\frac{\log _{10}(2 F A L)-1.51}{-0.0035}
$$

Donde la concentración de 2FAL es en ppm.

Este modelo fue renovado a partir de papel térmicamente mejorado (ver Ecuación 4). Este tipo de papel es el de mayor utilización en la actualidad por los fabricantes de transformadores de potencia.

$$
D P=\frac{\log _{10}(2 F A L \times 0.88)-4.51}{-0.0035}
$$

Donde la concentración de 2FAL es en ppb.

Ensayos fisicoquímicos del aceite: Estos ensayos son útiles para monitorear aceites en operación y permiten determinar el porcentaje de agua en el papel, el estado de degradación del aceite y el grado de impregnación de productos ácidos en el aceite. Ninguno de estos ensayos se debe tomar por si solo como un indicador confiable para tomar cualquier decisión en materia de mantenimiento, es necesario tener en cuenta todo el paquete de pruebas (IEEE C57.152, 2013; ASTM D3487, 2009).

Factor de potencia: Es una medida de las pérdidas de energía en forma de calor en el aislamiento durante la operación de un transformador (Naderian et al., 2009). El factor de potencia es un buen indicador de la calidad del aceite, así como de la condición del aislamiento de devanados, pasatapas, y pararrayos (IEEE C57.152, 2013).

Corriente de excitación: El propósito de este ensayo es detectar daños o cambios en la parte activa de un transformador (p.ej. espiras en cortocircuito de un devanado, láminas en cortocircuito de un núcleo, defectos en la estructura de un núcleo, etc). En la prueba de corriente de excitación se inyecta una tensión reducida en uno de los devanados con los terminales del otro devanado flotantes y se mide la corriente circulante (IEEE C57.152, 2013).

Relación de transformación: Este ensayo permite detectar espiras abiertas o en cortocircuito de un devanado, daños y posiciones incorrectas en el OLTC. Es recomendable realizar lecturas de relación de trasformación para todas las posiciones del OLTC (IEEE C57.152, 2013; IEEE C57.12.90, 2010).

Resistencia de devanados: Este ensayo permite detectar espiras abiertas o en cortocircuito de un devanado, conexiones sueltas y malos contactos en el OLTC. Es recomendable realizar lecturas de resistencia de devanados en la posición nominal, máxima y mínima del OLTC (IEEE C57.152, 2013; IEEE C57.12.90, 2010). 
Resistencia de aislamiento: Este ensayo es un indicador útil de contaminación y humedad en la superficie del aislamiento de un devanado. La resistencia de aislamiento es estimada a través de la relación entre la tensión aplicada durante el ensayo y la corriente circulante. (IEEE C57.152, 2013; IEEE C57.12.90, 2010).

Ensayos adicionales: Otros ensayos electromagnéticos de campo y de laboratorio son analizados con detalle en las normas IEEE C57.152 (2013) e IEEE C57.12.90 (2010).

Después de adquirir la información, el siguiente paso es diagnosticar la condición. Algunos de los procedimientos para diagnosticar la condición, se basan en establecer relaciones lineales entre los resultados de los diferentes ensayos realizados al transformador (Naderian et al., 2009). Este método, plantea que a cada resultado se le debe asignar un valor relativo y a cada prueba un peso específico de acuerdo a su importancia; luego se realiza por cada prueba el producto entre el valor relativo y el peso específico y finalmente se deben sumar todos los productos obtenidos; el valor resultante de la sumatoria corresponde a la condición del transformador evaluado. De esta forma, se puede clasificar su estado en función de la condición (Lara y Liñan, 2009). Sin embargo, esta técnica presenta dos desventajas: La primera es que los límites de aceptación entregados para los ensayos por la normatividad técnica internacional difieren entre si y además contienen un margen de incertidumbre, por lo tanto aparece una región difusa entre los limites. La segunda desventaja es que la asignación de pesos a las pruebas se basa en la experiencia, lo cual puede diferir entre un experto y otro. Esta desviación en los pesos, hace que el índice de salud estimado tienda a desviarse de un valor exacto (Abu Elanien et al., 2012).

Una solución para estos problemas es la utilización de lógica difusa. Esta técnica se basa en reglas de inferencia basadas en expresiones no numéricas (lingüísticas) (Ross, 2010), por lo tanto no se necesita un peso para cada prueba de diagnóstico. Para diseñar las reglas, es necesario identificar la criticidad de cada prueba y su relación con la condición del transformador. Para desarrollar una aplicación de lógica difusa, primero se deben fusificar las entradas, lo que significa convertir límites de aceptación numéricos en funciones lingüísticas, esto se denomina función de membresía. Por cada prueba de diagnóstico, deben identificarse funciones de membresía y estas pueden cruzarse entre sí, lo que significa que no se necesitan límites de aceptación exactos. Estas características de la lógica difusa superan las desventajas mencionadas de las relaciones lineales. En la literatura (Abu Elanien et al., 2012; Arshad e Islam, 2006; Ashkezari et al., 2013), realizan aplicaciones basadas en lógica difusa para estimar el índice de salud de un transformador.

\section{CONCLUSIONES}

La gestión de transformadores de potencia cobra cada día mayor importancia para las empresas, ya que los beneficios incluyen: mejoras en el rendimiento financiero, reducción de costos, información acerca de decisiones de inversión, mejoras en la gestión del riesgo, mejora de la continuidad y calidad del servicio, mejora de la eficiencia en el uso de activos, entre otros. Por lo tanto, para que las empresas puedan acceder a tales beneficios, es necesario que cuenten con sistemas eficientes de adquisición y almacenamiento de datos, que luego puedan ser procesados para generar información útil a los fines de la gestión del ciclo de vida del transformador, activo estratégico para la operación del sistema.

A partir de una revisión detallada del estado del arte, se presentaron los elementos o datos a considerar para la evaluación del riesgo y se introdujo al índice de riesgo del transformador, el cual constituye el elemento informativo que apoya al gestor del activo en el proceso de toma de decisiones, particularmente en aquellas relacionadas con las inversiones a realizar. El esquema para la valoración del riesgo, plantea resolver el problema desde dos puntos de vista: global e individual. El global, a través de la estimación del FC el cual considera factores estratégicos del sistema de potencia, como la ENS, el número de clientes afectados, el impacto sobre la seguridad del sistema, el impacto ambiental, etc. El individual, a través de la evaluación del PF basado en la condición, historia de operación, historial de fallas, entre otros aspectos.

Este documento sirve de guía para el desarrollo de una metodología para el cálculo del índice de riesgo del transformador, ya que organiza y describe los elementos que deben considerarse para tal fin. Se recomienda que los métodos que se implementen para la combinación de tales elementos consideren la experiencia de los operadores del sistema y del personal encargado del mantenimiento, operación y gestión del activo.

\section{REFERENCIAS}

Abu-Elanien A.B. y Salama M., Asset Management Techniques for Transformers, doi: 10.1016/j.epsr.2009.10.008, Electric Power Systems Research, 80(4), 456-464 (2010) 
Abu-Elanien A.B., Salama M., Ibrahim M., Calculation of a Health Index for Oil-Immersed Transformers Rated Under 69 kV Using Fuzzy Logic, IEEE Transactions on Power Delivery, 27 (4), 2029-2036 (2012)

Aponte G., Herrera W., González C. y Pleite J., Implementación de un Modelo de un Transformador Eléctrico para el Análisis de su Respuesta en Frecuencia, doi: 10.4067/S0718-07642011000400008, Inf. Tecnol., 22, (4), 59-72 (2011)

Arshad M., Islam S.M., A Novel Fuzzy Logic Technique for Power Transformer Asset Management, doi: 10.1109/IAS.2006.256536, Conference Record of the 2006 IEEE Industry Applications Conference Forty-First IAS Annual Meeting, 1, 276-286 (2006)

Ashkezari A., Hui M., Saha T., Ekanayake C., Application of fuzzy support vector machine for determining the health index of the insulation system of in-service power transformers, doi: 10.1109/TDEl.2013.6518966, IEEE Transactions on Dielectrics and Electrical Insulation, 20(3), 965-973 (2013)

ASTM D3487, American Society for Testing and Materials, Standard Specification for Mineral Insulating Oil Used in Electrical Apparatus, 1-6, United States (2009)

Bartley W. (Hartford Steam Boiler), Transformer Asset Management, https://www.hsb.com/TheLocomotive/ TransformerAssetManagement.aspx. Acceso 01 de noviembre (2014)

Bérubé J.N., Broweleit B.L. y Aubin J. (Electric Energy T\&D), Optimum Transformer Cooling Control with Fiber Optic Temperature Sensors, http://www.myvirtualpaper.com/doc/Electric-Energy/EE-MAG-JA2009/ 2009080501/\#54. Acceso 01 de noviembre (2014)

BSi PAS 55, British Standards Institution, Asset Management, London, United Kingdom, (2008)

Cardoso A.J. y Oliveira L.M., Condition monitoring and diagnostics of power transformers, International Journal of COMADEM , 2(3), 5-11, (1999)

Durán J., Gestión de Mantenimiento bajo Estándares Internacionales como PAS 55 Asset Management, Mantenimiento Mundial (en línea), http://www.mantenimientomundial.com/sites/mm/notas/PAS55.pdf. Acceso 01 de Noviembre (2014)

Energy D. (Office of Electricity Delivery and Energy Reliability), Large Power Transformers and the U.S. Electric Grid, Infrastructure Security and Energy Restoration, 1-52, United States (2014)

Eto J.H. y LaCommare K.H., Tracking the Reliability of the U.S. Electric Power System: An Assessment of Publicly Available Information Reported to State Public Utility Commissions, Berkley National Lab, 1-52, United States (2008)

Hardwick J., Aspects of Asset Management at Energy Australia, Energy Australia, 76th Annual International Conference of Doble Clients, Boston, United States (2007)

IEC 60300-2 ed2.0, International Electrotechnical Commission, Dependability management - Part 2: Guidelines for dependability management, 1-113, Ginebra, Suiza (2004)

IEC 60599, International Electrotechnical Commission, Mineral oil-impregnated electrical equipment in service. Guide to the interpretation of dissolved and free gases analysis, 1-74, Ginebra, Suiza (2007)

IEC 61198, International Electrotechnical Commission, Mineral insulating oils - Methods for the determination of 2-furfural and related compounds, 1-28, Ginebra, Suiza (1993)

IEEE C57.104, Institute of Electrical and Electronics Engineers, Guide for the Interpretation of Gases Generated in Oil-Immersed Transformers, 1-39, New York, United States (2008)

IEEE C57.12.90, Institute of Electrical and Electronics Engineers, Standard Test Code for Liquid-Immersed Distribution Power and Regulating Transformers, 1-100, New York, United States (2010)

IEEE C57.140-2006, Institute of Electrical and Electronics Engineers, Guide for the Evaluation and Reconditioning of Liquid Immersed Power Transformers, 1-100, New York, United States (2006) 
IEEE C57.152, Institute of Electrical and Electronics Engineers, Guide for Diagnostic Field Testing of FluidFilled Power Transformers Regulators and Reactors, 1-121, New York, United States (2013)

ISO 55000, International Standarization Organization, Asset management - Overview, Principles and Terminology, 1-19, Ginebra, Suiza (2014)

Kang P. y Birtwhistle D., Condition assessment of power transformer on-load tap-changers using wavelet analysis, doi: 10.1109/61.924817, IEEE Transactions on Power Delivery (en línea), 16(3), 394-400 (2001)

Lara H. y Liñan R., Clasificación del Estado Operativo de Transformadores de Potencia Mediante el Desarrollo e Implementación de una Metodología basada en los Resultados de Pruebas de Rutina, Congreso Internacional en Alta Tensión y Aislamiento Eléctrico, 1-6, Medellín, Colombia (2009)

Li W. Risk Assessment of Power Systems Models Methods and Applications, IEEE Press Series on Power Engineering, John Wiley \& Sons, 1-325 (2005)

Lundgaard L., Hansen W., Linhjell D., Painter T., Aging of oil-impregnated paper in power transformers, doi: 10.1109/TPWRD.2003.820175, IEEE Transactions Power Delivery, 19(1), 230-239 (2004)

Mariño P., Poza F., Ubeira M., Machado F., Sistema de Adquisición y Almacenamiento de Datos para Monitorización del Estado de Transformadores de Potencia, doi: 10.4067/S0718-07642004000200017, Inf. Tecnol. (en línea), 15, (2), 95-100 (2004)

Naderian A., Piercy R., Cress S., Service J., Fan W., An approach to power transformer asset management using health index, doi: 10.1109/MEI.2009.4802595, IEEE Electrical Insulation Magazine, 25(2), 20-34 (2009)

Reetz O., Asset Management Strategies to Preserve Operational Equipment, Magazine Energize (en línea), 26-29, 2005, http://www.ee.co.za/wp-content/uploads/legacy/Asset\%20management.pdf. Acceso 01 de Noviembre (2014)

Romero A.A., Mombello E.E., Ratta G., An overview on power transformer management: Individual Assets and Fleets, doi: 10.1109/TDC-LA.2012.6319081, Transmission and Distribution Latin America Conference and Exposition (T\&D-LA) Sixth IEEE PES (en línea), 1-7 (2012)

Ross T. (Wiley), Fuzzy logic with engineering applications, Third edition, 1-607, John Wiley \& Sons, New Mexico, United States (2010)

Shengchang J., Ping S., Yanming L, Dake X. y Junling C., The Vibration Measuring System for Monitoring Core and Winding Condition of Power Transformer, doi: 10.1109/ISEIM.2001.973811, Proceedings of 2001 International Symposium on Electrical Insulating Materials ISEIM 2001 (en línea), 849-852 (2001)

Sidwell N., Accuracy of furan analysis in estimating the degree of polymerization in power transformers, A research report submitted to the Faculty of Engineering and the Built Environment University of the Witwatersrand, 1-23 (2011)

Tichelman B., Transformer Asset Management - A new Paradigm (2004), http://www.energycentral. com/articles/article/678. Acceso 01 de noviembre (2014)

Wang M., Vandermaar A., Srivastava K., Review of condition assessment of power transformers in service, doi: 10.1109/MEI.2002.1161455, IEEE Electrical Insulation Magazine, 18(6), 12-25 (2002)

Zargari A, Blackburn T., Application of optical fibre sensor for partial discharge detection in high-voltage power equipment, doi: 10.1109/CEIDP.1996.564529, IEEE 1996 Annual Report of the Conference on Electrical Insulation and Dielectric Phenomena, 2, 541-544 (1996) 